\title{
Measuring Magnetic Fields from Water Masers Associated with a Synchrotron Protostellar Jet
}

\author{
Ciriaco Goddi ${ }^{1,2}$ and Gabriele Surcis ${ }^{3}$ \\ ${ }^{1}$ Department of Astrophysics/IMAPP, Radboud University, \\ P.O. Box 9010, 6500 GL Nijmegen, The Netherlands \\ ${ }^{2}$ ALLEGRO/Leiden Observatory, Leiden University, \\ PO Box 9513, NL-2300 RA Leiden, the Netherlands \\ email: cgoddi@strw.leidenuniv.nl \\ ${ }^{3}$ INAF - Osservatorio Astronomico di Cagliari \\ Via della Scienza 5 - I-09047 Selargius, Italy \\ email: surcis@oa-cagliari.inaf.it
}

\begin{abstract}
The Turner-Welch Object in the W3(OH) high-mass star forming complex drives a synchrotron jet, which is quite exceptional for a high-mass protostar, and is associated with a strongly polarized water maser source, $\mathrm{W} 3\left(\mathrm{H}_{2} \mathrm{O}\right)$, making it an optimal target to investigate the role of magnetic fields on the innermost scales of protostellar disk-jet systems. We report here full polarimetric VLBA observations of water masers. The linearly polarized emission from water masers provides clues on the orientation of the local magnetic field, while the measurement of the Zeeman splitting from circular polarization provides its strength. By combining the information on the measured orientation and strength of the magnetic field with the knowledge of the maser velocities, we infer that the magnetic field evolves from having a dominant component parallel to the outflow velocity in the pre-shock gas (with field strengths of the order of a few tens of $\mathrm{mG}$ ), to being mainly dominated by the perpendicular component (of order of a few hundred of $\mathrm{mG}$ ) in the post-shock gas where the water masers are excited. The general implication is that in the undisturbed (i.e. not-shocked) circumstellar gas, the flow velocities would follow closely the magnetic field lines, while in the shocked gas the magnetic field would be re-configured to be parallel to the shock front as a consequence of gas compression.
\end{abstract}

Keywords. stars: formation, ISM: jets and outflows, ISM: magnetic fields

\section{Introduction}

One relevant open question in high-mass star formation (HMSF) is the relation between the gas dynamics and the magnetic field in regulating mass-accretion and mass-loss. VLBI measurements of molecular masers can provide a detailed description of gas kinematics (Goddi et al. 2005, 2006, 2011, Matthews et al. 2010, Greenhill et al. 2013, Moscadelli \& Goddi 2014, Moscadelli et al. 2016, Issaoun et al. 2017) and magnetic field structure (Surcis et al. 2014, 2015, Sanna et al. 2015) on scales from tens to hundreds AU, which are the smallest accessible scales in studies of HMSF, and have therefore the potential to address such an open question. $\mathrm{W} 3\left(\mathrm{H}_{2} \mathrm{O}\right)$ contains the best known (archetypical) case of synchrotron jet driven by an embedded high-mass young stellar object or YSO (Reid et al. 1995) and associated with $\mathrm{H}_{2} \mathrm{O}$ masers, and therefore provides a good target for investigating this issue.

Here we summarize the main results from a full polarimetric study of $\mathrm{H}_{2} \mathrm{O}$ masers in $\mathrm{W} 3\left(\mathrm{H}_{2} \mathrm{O}\right)$. A full description of results is reported in Goddi et al. (2017). 

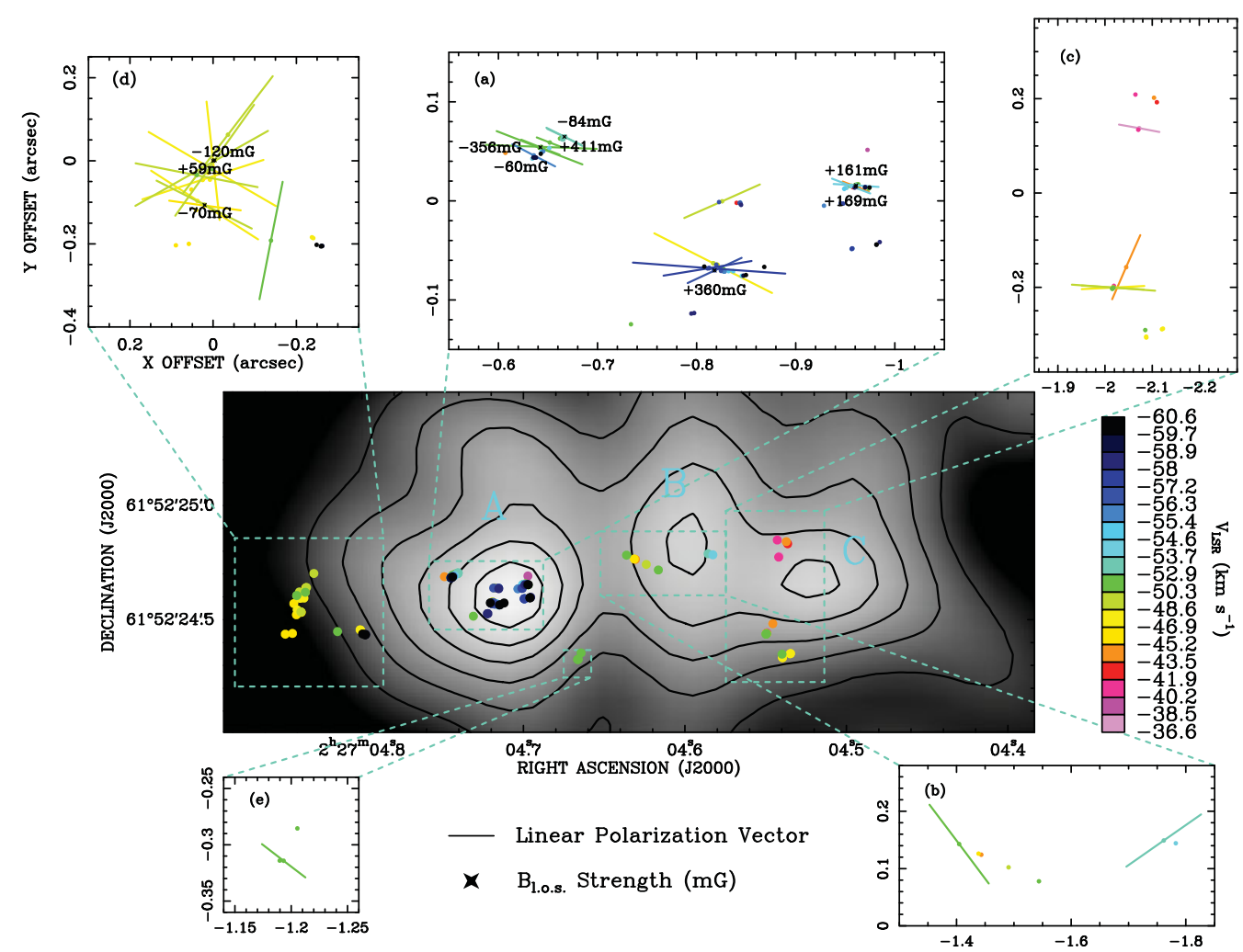

Figure 1. Overlay of the water masers detected with the VLBA in $\mathrm{W} 3\left(\mathrm{H}_{2} \mathrm{O}\right)$ onto the $1.4 \mathrm{~mm}$ continuum emission mapped with the PdBI by Wyrowski et al. (1999) (gray scale and black contours). The circles show positions of the $\mathrm{H}_{2} \mathrm{O}$ masers, while the colors denote their l.o.s. velocity in $\mathrm{km} \mathrm{s}^{-1}$ (color scale on the right-hand side). The three $1.4 \mathrm{~mm}$ continuum peaks are labelled "A", "B", and "C", from east to west. The insets show the linear polarization vectors of individual maser features in different clusters (from "a" to "e"), where the length of the line segments scales logarithmically with the polarization fraction (in the range $P_{1}=0.9 \%-42 \%$ ). We also report the magnetic field strengths (in $\mathrm{mG}$ ) along the l.o.s. ( $B_{\text {l.o.s. }}$ ) in the maser features for which we measured the Zeeman splitting. The positions are relative to the reference maser feature.

\section{Results}

We identified a total of 148 individual maser features and we measured their physical properties, including positions, flux densities, l.o.s. velocities $\left(\mathrm{V}_{\mathrm{LSR}}\right)$, and (when polarized) their fraction of linear and circular polarizations, as well as the corresponding linear polarization angles and magnetic field strengths along the l.o.s.. The methodology adopted to derive the physical properties of individual maser features is described in Appendix A in Goddi et al. (2017).

Out of the 148 features detected, we measured linear polarization in 34 maser features, with a fractional percentage varying in the range $P_{1}=0.9 \%-42 \%$. In Figure 1 , line segments indicate the linear polarization vectors of the maser features, whose length scales logarithmically with the polarization fraction, $P_{l}$. We established an empirical threshold of linear polarization fraction $\left(P_{1}=5 \%\right)$ above which the water masers enter into the saturation regime, and their polarized signal does not trace the magnetic field anymore (see Goddi et al. 2017 for details). Figure 2 shows the resulting (sky-projected) magnetic field vectors for the maser features with $P_{1}<5 \%$, overplotted on the $1.4 \mathrm{~mm}$ 


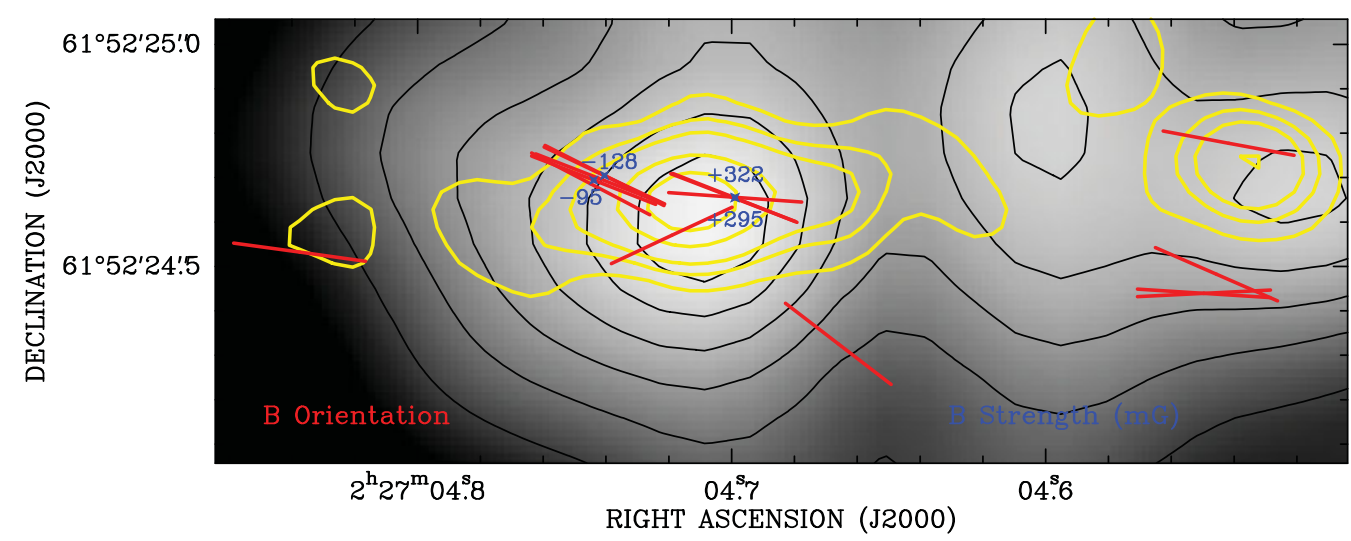

Figure 2. Magnetic field orientation (in the plane of the sky) for 17 individual masers with $P_{l}<5 \%$ (red segment) and strength for 4 (non-saturated) masers for which the Zeeman splitting was measured. The $8.4 \mathrm{GHz}$ emission imaged with the VLA (beamsize $0^{\prime \prime} 2$ ) by Wilner et al. (1999) (yellow contours) is overploted onto the $1.4 \mathrm{~mm}$ continuum emission mapped with the PdBI (beamsize 0." 5 ) by Wyrowski et al. (1999) (gray scale and black contours: same as in Fig. 1).

continuum map tracing the dust emission (same as in Figure 1), as well as the $8.4 \mathrm{GHz}$ continuum imaged with the VLA (Wilner et al. 1999), tracing the synchrotron emission. Besides linear polarization, we also detected circularly polarized emission toward ten maser features, varying in the range $P_{\mathrm{V}}=0.2-1.6 \%$. Considering only masers with $P_{1}<5 \%$, we estimated magnetic field strengths of $\sim 100-300 \mathrm{mG}$ (see Figure 2).

\section{Discussion}

The magnetic field inferred from the $\mathrm{H}_{2} \mathrm{O}$ masers is on average oriented along $\mathrm{E}-\mathrm{W}$ (Figure 2), well aligned with the axis of the synchrotron jet, suggesting that the molecular masers may probe the magnetic field in the protostellar jet. Figure 3 shows an overlay of the dust emission (greyscale) from the hot-core (Wyrowski et al. 1999), synchrotron emission (white contours) from the radio jet (Wilner et al. 1999), water maser proper motions (arrows) measured by Hachisuka et al. 2006, and the direction of magnetic field vectors as determined from our polarization measurements (purple segments). This overlay reveals a misalignment between the magnetic field and the velocity vectors, which can be explained with an origin in magnetically supported shocks. In particular, the shock passage alters the initial magnetic field configuration in the circumstellar gas, by compressing and enhancing the component of the magnetic field perpendicular to the shock velocity with respect to the parallel component (by a factor equal to the ratio between the post- and pre-shock densities: typically a 100). In the gas shocked by the synchrotron jet, we estimate a total field strength in the range $\sim 100-300 \mathrm{mG}$ (at densities of $10^{9} \mathrm{~cm}^{-3}$ ). Although the water maser polarization measurements alone cannot provide a direct measurement of the magnetic field properties in the quiescent (preshock) circumstellar gas, by combining the information on the orientation and strength of the magnetic field (in the post-shock gas) with the knowledge of the maser velocities, we are able to constrain the magnetic field strength in the pre-shock circumstellar gas (at densities of $10^{7} \mathrm{~cm}^{-3}$ ) to 10-20 mG (see Goddi et al. 2017 for details). 


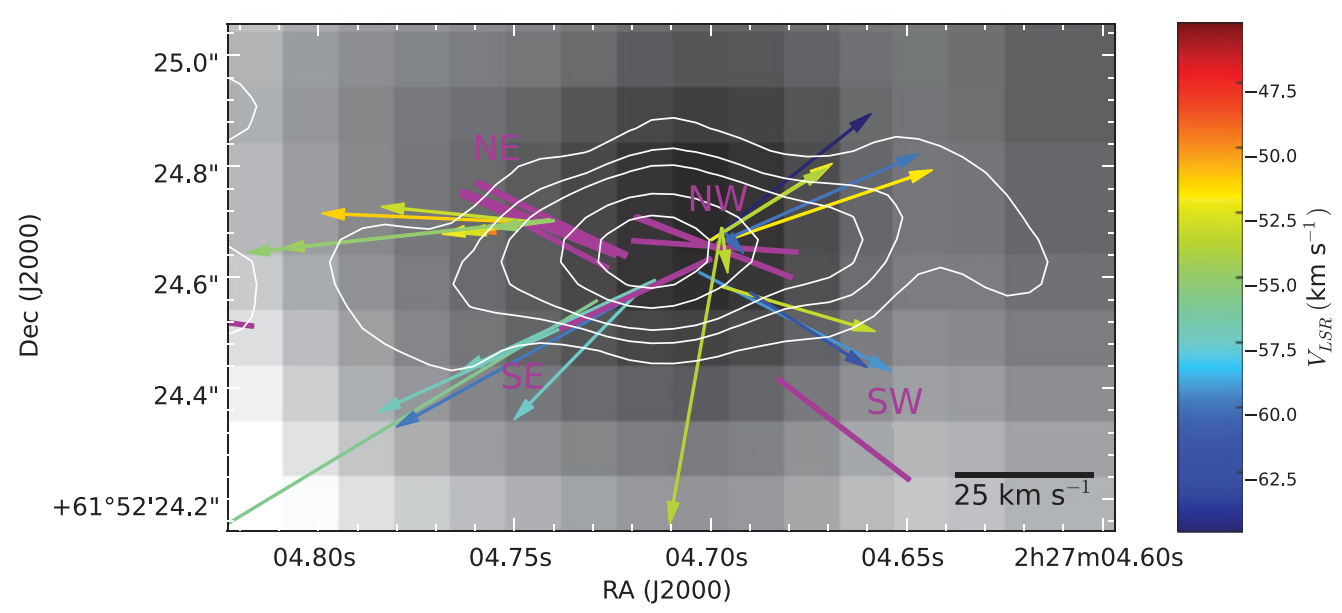

Figure 3. Comparison between the magnetic field orientations of $\mathrm{H}_{2} \mathrm{O}$ masers (purple segments) and proper motions of $\mathrm{H}_{2} \mathrm{O}$ masers (arrows). The $8.4 \mathrm{GHz}$ emission imaged with the VLA by Wilner et al. (1999) (white contours) is overploted onto the $1.4 \mathrm{~mm}$ continuum emission mapped with the PdBI by Wyrowski et al. (1999) (gray scale). Colors denote l.o.s. velocity (color scales on the right-hand side) and the scale for the proper motion amplitude is given in the lower right corner (both in $\mathrm{km} \mathrm{s}^{-1}$ ). There are four main knots of masers, located towards NE, NW, SE, and SW, with respect to the radio continuum peak. The proper motions identify a biconical, bipolar molecular outflow. Note the misalignment between magnetic field and velocity vectors, particularly in the NE and NW knots.

\section{Conclusions}

Our results in $\mathrm{W} 3\left(\mathrm{H}_{2} \mathrm{O}\right)$ suggest the presence of a local coupling between the magnetic field and the gas kinematics, indicating that magnetic fields can be dynamically important in driving the gas outflowing from a high-mass protostar.

\section{References}

Goddi, C., Moscadelli, L., Alef, W., et al. 2005, A\&\&A, 432, 161

Goddi, C., Moscadelli, L., Torrelles, J. M., Uscanga, L., \& Cesaroni, R. 2006, A\& A, 447, L9

Goddi, C., Moscadelli, L., \& Sanna, A. 2011, A\& A, 535, L8

Goddi, C., Surcis, G., Moscadelli, L., et al. 2017, A\& A, 597, A43

Greenhill, L. J., Goddi, C., Chandler, et al. 2013, ApJ (Letters), 770, L32

Hachisuka, K., Brunthaler, A., Menten, K. M., et al. 2006, ApJ, 645, 337

Issaoun, S., Goddi, C., Matthews, L. D., et al. 2017, A\&A, 606, 126

Matthews, L. D., Greenhill, L. J., Goddi, C., et al. 2010, ApJ, 708, 80

Moscadelli, L. \& Goddi, C. 2014, A\& A, 566, A150

Moscadelli, L., Sánchez-Monge, Á., Goddi, C., et al. 2016, A\&̊A, 585, A71

Reid, M. J., Argon, A. L., Masson, C. R., Menten, K. M., \& Moran, J. M. 1995, ApJ, 443, 238

Sanna, A., Surcis, G., Moscadelli, L., et al. 2015, A\&A, 583, L3

Surcis, G., Vlemmings, W. H. T., van Langevelde, H. J., et al. 2014, A\&A, 565, L8

Surcis, G., Vlemmings, W. H. T., van Langevelde, H. J., et al. 2015, A\& A, 578, A102

Wilner, D. J., Reid, M. J., \& Menten, K. M. 1999, ApJ, 513, 775

Wyrowski, F., Schilke, P., Walmsley, C. M., \& Menten, K. M. 1999, ApJ (Letters), 514, L43 